\title{
Assistive Technologies: Addressing the Divide Between the Developed and Developing World
}

\section{Stuti Chakraborty}

Christian Medical College, Vellore, Department of Physical Medicine and Rehabilitation, Christian Medical

College and Hospital, Ida Scudder Road, Vellore, Tamil Nadu - 632004, India

https://doi.org/10.38126/ISPG 160201

Corresponding author: stutibb@gmail.com

Keywords: assistive technologies; low income countries; lower-middle income countries; high income countries; UNCRPD; people with disabilities

Executive Summary: Assistive technologies are used to enhance the day-to-day functionality of people with disabilities by improving their quality of life and by reducing the impact of disability in their lives. Various socio-economic, cultural, contextual, medical, personal, and family-related factors determine the feasibility and outcomes of using assistive technologies. While various forms of assistive technologies are being widely used for rehabilitation, recreational, or personal purposes around the world many still remain inaccessible and vastly unaffordable in countries around the world. Literature suggests that, when comparing high income countries with low and lower-middle income countries, differences among the longevity, availability, ease of users, affordability and, most importantly, the recognition of the need for an assistive technology, are common, with those measures lagging in low and lowermiddle income countries. Certain targeted interventions have been suggested to provide insight into how discrepancies in promoting the use of assistive technologies between developed and developing nations can be minimised with the goal of reducing the global impacts of disability.

\section{Introduction}

Assistive technologies can be broadly considered as "any item or piece of equipment or product system, whether acquired commercially, off-the-shelf, modified or customised, that is used to increase, maintain or improve functional capabilities of people with disabilities" (Assistive Technology Act 2004; Bausch et al. 2005). In the context of healthcare specifically, the use for assistive technologies is widespread and can also be referred to as health state assistive technology devices. These devices can be either intrinsic or extrinsic. Intrinsic devices are implanted and optimise functioning by replacing a body function of body structure such as cochlear implants, cardiac pacemakers. Extrinsic devices are not implanted and optimise function by augmenting, restoring, or compensating for body function, and structure impairments, such as wheelchairs, glasses, hearing aids, or augmentative and alternative communication devices. Both extrinsic and intrinsic assistive technologies are used extensively to reduce the impact of disability (Bauer et al. 2011).

The World Health Organisation (WHO) estimates that there are around one billion people living with disabilities worldwide. Increasing incidences of non-communicable diseases will cause more than two billion people globally to use at least one assistive technology product. (World Health Organisation 2018). The World Bank defines a low income country (LIC) as having a gross national income (GNI) per capita of US\$1,025 or less, a lower-middle income country (LMIC) as having a GNI per capita between US $\$ 1,026-$ US $\$ 3,995$, and a high income country (HIC) as having a GNI per capita of US $\$ 12,376$ or more (Prydz and Wadhwa 2019). Almost $80 \%$ of people with disabilities in 
lower-middle income countries experience difficulty accessing rehabilitative services and could potentially benefit from increased use or availability of assistive technology. However, this estimate cannot be considered comprehensive as it does not include different types of assistive technologies such as wheelchairs or devices aiding in communication and vision, nor does it take into consideration people with disabilities using more than one form of assistive technology (Borg, Lindström, and Larsson 2011; World Health Organisation 2011; Matter et al. 2017; Prydz and Wadhwa 2019).

\section{Assistive technologies in the context of the United Nations Convention on the Rights of Persons with Disabilities}

The United Nations Convention on the Rights of Persons with Disabilities (UNCRPD) entitles its beneficiaries to access to assistive technologies to ensure full and equal enjoyment of all fundamental freedoms and requires governments to act upon to enforce the same (United Nations General Assembly 2006). The implementation of the UNCRPD, however, remains far from reality. Once an assistive technology has been obtained by the user, it is imperative to ensure its proper maintenance and operation by making the user familiar with its functionality. This can be done by addressing various factors ranging from an extensive assessment of individual needs, correct fit, device selection, and training and follow-up to ensure safety and efficiency while in use. If these factors are not given the importance they require, it can often result in the targeted need for which the assistive technology was initially adopted not being met. Follow-up after getting an assistive technology has been found to be significantly lacking in LICs and LMICs when compared to HICs. This is one of the major contributing factors behind the lopsided implementation of the UNCRPD at a global level.

Environmental barriers that hinder accessibility are a major cause for using certain types of assistive technology when it comes to LICs and LMICs. According to the International Classification of Functioning, Disability and Health, environmental factors "refer to all aspects of the external or intrinsic world that form the context of an individual's life and, as such, have an impact on the person's functioning. Environmental factors include the physical world and its features, the humanmade physical world, other people in different relationships and roles, attitudes and values, social systems and assistive technologies, and policies, rules and laws" (World Health Organisation 2001). Barriers are defined as "environmental factors in a person's environment that, through their absence or presence, limit functioning and create disability". (World Health Organisation, 2001).

\section{Causes and considerations of limited usage in LMICs and LICs}

There are several reasons contributing to the lack of recognition of need and usage of assistive technologies in the developing world. Recent reviews have demonstrated that research regarding assistive technology use especially in LICs or LMICs has received limited attention (Matter et al. 2017). In addition, the varied and broad categories of assistive technologies are not being researched adequately, especially in LMICs where the majority of importance is given only to leg prosthesis and wheelchair use. Literature suggests that there is negligible research supporting the efficacy and feasibility of use for other types of assistive technologies such as those used for aural or cognitive rehabilitation. This lack of research also poses as a roadblock for the effective implementation of UNCRPD and needs for assistive technologies in LMICs. Furthermore, the unavailability of detailed knowledge, despite the presence of a broad awareness of the situation, hinders the process of policymaking and allocation of resources to ensure the efficient use of assistive technologies by people living in LMICs.

Another factor to be considered is the age group of the demographic population in need of assistive technologies. Functional limitations restricting participation in activities due to impairment and disability among the elderly in LMICs are expected to be much higher than in higher income countries. Although some LMICs such as India, Cambodia, Egypt, and Brazil currently have various assistive technology initiatives in production at lower costs, there are still many LMICs where either the production is low or costs are too expensive.

Studies have also demonstrated a "service delivery gap" in relation to the working conditions of assistive technologies supplied with instructions for 
use. Maintenance and post-delivery services are largely left to the owner and their family. Literature also suggests that programs pertaining to policies for assistive technologies were majorly skewed towards serving people living in higher income countries. For example, the assistive technologies policy program in Norway enables persons in need of assistive technologies to receive financial assistance in their procurement under the national insurance scheme (Borg, Lindström, and Larsson 2011; Boger et al. 2017; Rohwerder 2018). Under the national insurance scheme, assistive technologies are available for stimulation, training, and activation of children and young people under the age of 26. It also considers the inclusion of provision of other means of assistance such as guide dogs, reading and secretarial assistance for people with impaired vision, and computer software to train and serve as a mode of communication for young adults and children (Sund 2017).

A report published by the World Federation of Occupational Therapists' recently highlighted that provision of assistive technologies met the needs of individuals well in HICs, whereas the same needs were met poorly in LICs or LMICs (World Federation of Occupational Therapy 2019). While fields of engineering have advanced immensely and contributed significantly to medical rehabilitation, people with disabilities in LICs and LMICs still lag behind those in HICs when it comes to accessing benefits of these technologies. Another significant cause of this divide amongst HICs and LMICs appears in the differing accessibility based on gender. Gender disparity in terms of use and accessibility of assistive technologies has also been shown where there are more men using assistive technologies than women in LMICs, such as Zimbabwe and Namibia. (MacLachlan and Swartz 2009)

\section{Recommendations requiring immediate deliberation and intervention by LMICS and LICs}

i. Reinforcing the role of rehabilitation professionals

Providing an individual with the correct assistive technology can immensely impact the outcome of long-term rehabilitation and adequate translation of the skills acquired by the patient to use the particular assistive technology in different contexts. Rehabilitation professionals who provide these services must ensure the correct prescription is given, facilitate informed decision making, and enable people with disabilities to choose a particular type of assistive technology while keeping in mind the overall goal of improving quality of life for that individual.

Often, developing countries lag behind when it comes to the ability of people with disabilities to gain access to assistive technology due to the stigma associated with disability. While persistent across the globe, stigma continues to be a challenge more deep-rooted and severe in developing countries. A major chunk of rehabilitation constitutes ensuring that the client is able to use the assistive technology in various contexts whether social, physical, or personal and empowering the individual to be able to effectively cope with the stigma associated with using an assistive technology. These challenges must be addressed to the maximum extent possible by rehabilitation professionals at an individual level through early identification and elimination of factors such as stigma, doubt, or fear during the process of rehabilitation, for the person with disabilities as those around them. When suggesting an aid, professionals must be aware of the client's functional capacity, level of motivation to use the aid, educational, environmental, psychological, and socio-economic status in order to enable the client to efficiently make full use of the assistive technology (Williams et al. 2017; Kristiansen 2018).

Professionals working in LICs must also be mindful when suggesting aids to clients from lower socioeconomic backgrounds and look for suitable, yet more affordable, versions of equipment at lower and subsidised costs. Sensitisation of communities in LICs through methods such as health education workshops, health campaigns, and diversity appreciation activities, especially in rural areas, can help address the stigmatisation of people with disabilities using aids and appliances. Governmentapproved rehabilitation councils and bodies of governance should outline the professional requirements of rehabilitation personnel as well as provide training, education, licensing, and establishing accountability for rehabilitation professionals could benefit the practice of rehabilitation but such systems are currently in place in only a few LICs and LMICs. 
ii. Eradicating the importance hierarchy and treating all forms of disability equally

While adaptive devices have been automated to a certain extent in the developing world, very little exploration of the use of technology to assist people with disabilities facing cognitive, hearing, or visual difficulties has taken place. One of the probable reasons for this could be a hierarchy of importance assigned to various forms of disability. Cognitive or visual deficits are equally viewed as disabling conditions in HICs, the same might not always be the case in LICs or in LMICs (Boger, et al. 2017).

A way to address this would be to encourage and empower more people with visual, hearing or cognitive disabilities to come forward as examples of change, social leaders, and role models in order to increase awareness about living with disabilities other than a physical one through engagement in various self-help groups or disabled peoples' organisations. While people with disabilities can act as role models, the onus for disability awareness cannot be the sole responsibility of people with disabilities. Local, regional and national level authorities along with the support of respective communities need to be equally involved in the process. This could be done by through the incorporation of people with disabilities and into mainstream society through media outreach and entrusting them with leadership roles in politics, business, academia, or other fields. Governments must also focus on investing in the research and development of practical, context-appropriate and viable forms of assistive technology that cater specifically to underrepresented classes of disabilities.

iii. Centralisation of resource distribution and adoption of a multi-sectoral approach

Centralisation of resources within a single entity to examine, supply and reproduce assistive technologies at a national level can be more effective than regional distribution and reduce chances of misinformation or incorrect data handling, thereby ensuring appropriate allocation of and accountability for funding. Apart from this, advocating for the reform of existing policies or proposing new policies to support a positive shift towards overcoming the demand-supply shortage could be one of the fundamental steps towards acquiring centralisation.
An example of best practices can be taken from Norway, a high income country, which has established a Central Unit of Purchases responsible for placing purchase orders for all assistive devices, including spare parts, accessories, and services (except technical services) from dealers on behalf of the assistive technology centers. Moreover, it is imperative to adopt a transdisciplinary approach involving multiple stakeholders such as developers, distributors authorities, academia, and most importantly, the users themselves to support the development of assistive technologies which are responsive to user needs and commercially viable. (Sund 2017).

iv. Addressing disparities between urban and rural areas

Even HICs such as the United States see significant variations in the accessibility of assistive technologies and presence of experienced personnel to provide them across urban and rural areas, especially affecting the elderly community. (Whitacre 2016; Weden et al. 2018). One of the ways to address the urban-rural divide in terms of accessibility can be through extensive communitybased rehabilitation models. Community health workers who carry out a major chunk of community based rehabilitation play a pivotal role in assessing the need for assistive technologies for people with disabilities based on their environmental, socioeconomic, or familial contexts. Thus, they play a pivotal role in bridging the gap between rural communities, hospitals, available government schemes, or provisions and utilisation rehabilitation measures targeting physical or mental health. Mapping out of territories with least access due to environmental or attitudinal barriers could serve as a means of keeping track of assistive technology utilisation thereby preventing their overuse and supply in certain regions more than the other and ensure equitable distribution of government-funded technologies.

\section{v. Treating each individual client holistically}

The use of assistive technologies is varied and not limited to people with disabilities. Assistive technologies can be used extensively for geriatrics, paediatrics, neuromusculoskeletal injuries, and mental illness, just to name a few. Apart from the healthcare sector, they can also be helpful in other sectors such as education or administration to 
enhance productivity. It is essential to address each client's psychological and emotional status while prescribing assistive technologies to facilitate a smooth transition and increase the client's adaptability to the prescribed technology in various environmental contexts relevant to the client. Language, culture, traditional beliefs and values, role expectations of the client from family and society, and legal limitations are also factors that could potentially influence a client's readiness to use an assistive technology. Various barriers to the adoption of assistive technologies by older people addressed through a systematic review outlined privacy and trust to be their main concerns (Yusif S. et. al 2016).

Another key barrier is the ability of the client to use it functionally in day-today life with ease. Most importantly, clients must understand their own needs how assistive technologies may improve their quality of life while also understanding that having the assistive technology will not completely ameliorate the disability, though it may improve their quality of life. Caregiver engagement through support groups could also potentially intensify and catalyse the realisation of appropriate use of assistive technology when coupled with opportunities to share about their experiences of being a caregiver of an individual with disability (Tangcharoensathien et al. 2018).

\section{Conclusion}

Assistive technologies could be a life-long companion for people with disabilities and have an

\section{References}

Assistive Technology Act, 29 U.S.C (2004). Accessed: 16 May 2020.

https://www.govtrack.us/congress/bills/108/ hr4278/text

Bausch, Margaret E., Joel E. Mittler, Ted S. Hasselbring, and Donald P. Cross. 2005. "The Assistive Technology Act of 2004: What Does It Say and What Does It Mean?." Physical Disabilities: Education and Related Services 23, no. 2: 59-67.

Borg, Johan, Anna Lindström, and Stig Larsson. 2011. "Assistive technology in developing countries: a review from the perspective of the Convention on the Rights of Persons with Disabilities." Prosthetics and Orthotics International 35, no. 1: 20-29. https://doi.org/10.1177/0309364610389351 immense positive impact on their quality of life, provided it is the right fit for the individual. Having better access to various forms of assistive technologies would enable a huge proportion of people with disabilities who are currently dependent on caregivers for performing their daily activities and jobs to increase their independence, which could be an immense boost to the productivity of various developing nations. Adequate emphasis on and effective implementation measures taken to reinforce the role of rehabilitation professionals, eradication of hierarchies of importance for assistive technologies, centralisation of resource distribution while adopting a transdisciplinary approach, reduction of disparities amidst urban and rural areas, as well as adoption of a holistic approach to client treatment could create a sustainable impact and catalyse successful community reintegration.

While developed countries lead significantly in the community reintegration of people with disabilities owing to certain mechanisms in place already to necessitate a mass outreach of assistive technology, developing countries still lag behind, attributable to several factors as discussed above. Thus, it is essential to address this disparity urgently with the collaborated involvement of stakeholders across all levels in order to facilitate attainment of universal health coverage.
Boger, Jennifer, Piper Jackson, Maurice Mulvenna, Judith Sixsmith, Andrew Sixsmith, Alex Mihailidis, Pia Kontos, et al. 2017. "Principles for fostering the transdisciplinary development of assistive technologies." Disability and Rehabilitation: Assistive Technology 12, no. 5: 480-490. https://doi.org/10.3109/17483107.2016.1151 $\underline{953}$

Kristiansen, Lisbeth. 2018. "Wanting a Life in Decency!: A Qualitative Study from Experienced Electric Wheelchairs Users' perspective." Open Journal of Nursing 8, no. 7: 419-433. https://doi.org/10.4236/ojn.2018.87033 
MacLachlan, Malcom and Leslie Swartz, ed. 2009. Disability \& International Development: Toward Inclusive Global Health. New York: Springer.

Matter, Rebecca, Mark Harniss, Tone Oderud, Johan Borg, and Arne H. Eide. 2017. "Assistive technology in resource-limited environments: A scoping review." Disability and Rehabilitation: Assistive Technology 12, no. 2: 105-114. https://doi.org/10.1080/17483107.2016.1188 $\underline{170}$

Rohwerder, Brigitte. 2018. Assistive Technologies in Developing Countries. K4D Helpdesk Report. https://opendocs.ids.ac.uk/opendocs/handle/2 $\underline{0.500 .12413 / 13599}$

Sund, Terje, ed. 2017. Assistive Technology in Norway - a Part of a Larger System. Norway Department of Assistive Technology.

https://www.nav.no/en/home/aboutnav/publications//attachment/download/7b1 19b1c-fe72-488a-a1efbe424e72faff:c52b8c6ee759299749538a6fd05 54d1efa695abf/assistive-technology-innorway-170217v2.pdf

Prydz, Espen Beers and Divyanshi Wadhwa. 2019. "Classifying countries by income." The World Bank. Accessed: May 16, 2020.

https://datatopics.worldbank.org/worlddevelopment-indicators/stories/theclassification-of-countries-by-income.html

Tangcharoensathien, Viroj, Woranan Witthayapipopsakul, Shaheda Viriyathorn, and Walaiporn Patcharanarumol. 2018. "Improving access to assistive technologies: challenges and solutions in low-and middle-income countries." WHO South-East Asia Journal of Public Health 7, no. 2: 84-89. https://doi.org/10.4103/2224-3151.239419

United Nations General Assembly. 2006. Convention on the Rights of Persons with Disabilities, A/RES/61/106 (13 December 2006).

Weden, Margaret M., Regina A. Shih, Mohammed U. Kabeto, and Kenneth M. Langa. 2018. "Secular trends in dementia and cognitive impairment of US rural and urban older adults." American Journal of Preventive Medicine 54, no. 2:164-172.

Whitacre, Brian, 2016. "Technology Is Improving - Why Is Rural Broadband Access Still a Problem?" The Conversation. Accessed April 19, 2020.

http://theconversation.com/technology-isimproving-why-is-rural-broadband-access-stilla-problem-60423
Williams, Emma, Elizabeth Hurwitz, Immaculate Obaga, Brenda Onguti, Adovich Rivera, Tyrone Reden L. Sy, R. Lee Kirby, et al. 2017. "Perspectives of basic wheelchair users on improving their access to wheelchair services in Kenya and Philippines: a qualitative study." $B M C$ International Health and Human Rights 17, no. 1: 22. https://doi.org/10.1186/s12914-017$\underline{0130-6}$

World Federation of Occupational Therapists (WFOT). 2019. Global Indicators of Assistive Technology Use amongst Occupational Therapists: Report from the World Federation of Occupational Therapists' (WFOT) Global Surveys. WFOT. Accessed: 16 May 2020

https://www.wfot.org/resources/globalindicators-of-assistive-technology-useamongst-occupational-therapists-report-ofwfots-global-surveys

World Health Organisation. 2001. International Classification of Functioning, Disability and Health: ICF. Geneva: World Health Organisation. Accessed May 16, 2020.

https://apps.who.int/iris/bitstream/handle/10 665/42407/9241545429.pdf

World Health Organisation. 2002. Towards A Common Language for Functioning, Disability and Health: ICF. Geneva: World Health Organisation. Accessed: May 16, 2020. https://www.who.int/classifications/icf/icfbeg innersguide.pdf

World Health Organisation. 2011. World Report on Disability 2011. Geneva: World Health Organisation, Accessed: May 16, 2020.

https://apps.who.int/iris/bitstream/handle/10 665/44575/9789240685215 eng.pdf

World Health Organisation. 2018. "Assistive Technology Factsheet." WHO Accessed: 16 May 2020. https://www.who.int/news-room/factsheets/detail/assistive-technology

Yusif, Salifu, Jeffrey Soar, and Abdul Hafeez-Baig. 2016. "Older people, assistive technologies, and the barriers to adoption: A systematic review." International Journal of Medical Informatics 94: 112-116. https://doi.org/10.1016/j.ijmedinf.2016.07.00 $\underline{4}$

Stuti Chakraborty is currently an intern at Christian Medical College, Vellore. She is also a country representative for Healthcare Information for All; a country correspondent for YourCommonwealth; a member of the Global Healthcare Workforce Network and a country correspondent for IHP Global. She 
recently started working with Global Health Mentorships as a content facilitator and has also been a pioneer member for the launch of the India Chapter of Women in Global Health as a Chapter Officer over the last few months. In her work, she advocates for young people's health with a special focus on the rights of people with disabilities. She wishes to pursue research in the field of neurosciences in the near future.

\section{Acknowledgements}

The author would like to thank Mr. Abhishek Chandramouli who pursued his Bachelor of Technology degree in Computer Science and Engineering from Vellore Institute of Technology, Vellore and is currently doing research at the Massachusetts Institute of Technology Media Lab. 